Methods: Retrospective single-center observational study conducted in a Spanish tertiary hospital. In patients with high clinical suspicion of IIM, a myositis immunoblot was performed including Jo1, PL-7, PL-12, EJ, SRP, Mi2, Ku, MDA-5 TIF1- $\gamma$, HMGCR, PM-Scl and Ro52 antibodies. The demographic characteristics, the risk of ILD, cancer and death were analyzed.

Results: In a cohort of 313 patients with high suspicion of IIM, 87 patients $(27.8 \%)$ presented a positive MSA (MSA+ve). The mean age at diagnosis was $56.7 \pm 16.9$ years, with no significant differences between MSA+ve and MSA-ve $(p=0.597)$. Most of the patients were women with significant differences between both groups (80.5\% MSA+ve vs $68.1 \%$ MSA-ve, $p=0.030)$.

IIM were classified as antisynthetase syndrome (ARS) (38\%), dermatomyositis (DM) $(31 \%)$, overlap myopathy (OM) $(16.9 \%)$ and necrotizing myopathy (NM) (14.1\%) according to the manifestations and MSA found (Jo1, PL-12, PL-7, EJ in ARS; Mi-2, MDA-5 and TIF1- $\gamma$ in DM; Ku and PM-Scl in OM; HMGCR and SRP in NM). The most frequent MSA were anti-Jo1 (16.9\%), TIF1- $\gamma(15.5 \%), \mathrm{Ku}$ (12.7\%), Mi-2 (9.9\%), PL-7 (9.9\%), HMCGR (8.5\%), PL-12 (7\%), MDA-5 (5.6\%), SRP $(5.6 \%)$ and EJ $(4.2 \%)$. The presence of Ro52 associated with other MSA was found in 20 patients $(22.9 \%)$

ILD was the most frequent manifestation ( $45.2 \%$ of the MSA+ve). Non-specific interstitial pneumonia (NSIP) was the most frequent ILD $(39.5 \%)$, followed by usual interstitial pneumonia (34.2\%). The main risk factors associated with IIM-ILD were some subtypes of the MSAs $(p<0.001)$, the association of Ro52 $(p<0.001)$, and older age $(p=0.027)$. Among the IIM, ARS and OM $(p<0.001)$ were more frequently associated with IIM-ILD. The MSAs most associated with IIM-ILD were Jo1, PL-7, PM-Scl, Ku and SRP $(p<0.001)$

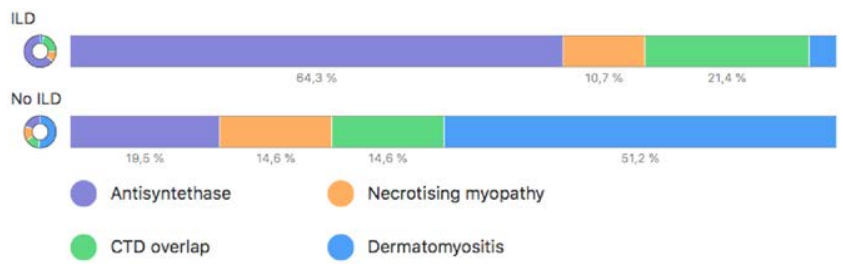

Cancer was found in $9.6 \%$ of MSA+ve patients. The most frequent tumors were gynecological (37.5\%), followed by gastrointestinal $(25 \%)$ and breast cancer $(12.5 \%)$. Factors associated with cancer were age $(p=0.010)$, TIF $1-\gamma(p<0.001)$, $\operatorname{SRP}(p=0.004), P L-12(p=0.013), P L-7(p=0.047)$ and HMGCR $(p=0.027)$.

The mortality of these patients was $3.5 \%$. There were no differences regarding MSA+ve/-ve $(p=0.911)$. However, MDA-5 $(p=0.033)$ and older age $(p=0.001)$ were associated with higher mortality. There were no significant differences between the IIM classifications, the associated SAD, the presence of cancer or ILD. However, longer follow-up periods and future studies are necessary to confirm these results.

Conclusion: The use of a myositis blot allowed classifying, stratifying the risk of ILD, the risk of cancer and the risk of mortality in IIM. IIM-ILD was the most frequent complication, usually manifested as NSIP. The associated risk factors were ARS, OM, some MSAs, Ro52+ and older age. Cancer was a serious and frequent manifestation in these patients, especially in patients with TIF1- $\gamma$ and other MSAs, so it is essential to know the risk factors and perform an early screening, especially in older patients.

A better knowledge of the serological profiles of IIM will provide more individualized approaches and better risk stratification, helping in the management and treatment of these patients.

REFERENCES:

[1] Satoh et al. Clin Rev Allergy Immunol. 2017 Feb;52(1):1-19

[2] Betteridge et al. J Intern Med. 2016 Jul;280(1):8-23.

Disclosure of Interests: None declared

DOI: 10.1136/annrheumdis-2021-eular.3234

\section{POS0876 \\ SARCOPENIA IN MYOSITIS PATIENTS: A MARKER OF MUSCLE DAMAGE ASSOCIATED WITH HANDICAP}

M. Giannini ${ }^{1}$, A. L. Charles ${ }^{2}$, M. Pizzimenti ${ }^{3}$, L. Debrut ${ }^{3}$, D. A. Levy ${ }^{1}$, R M. Javier ${ }^{4}$, B. Geny ${ }^{3}$, A. Meyer ${ }^{1}{ }^{1}$ University of Strasbourg, Service de Physiologie et d'explorations fonctionnelles, Nouvel Hopital Civil, Centre de Référence des Maladies Autoimmunes Rares, Fédération de Médecine Translationnelle, UR3072, Strasbourg, Strasbourg, France; ${ }^{2}$ University of Strasbourg, Fédération de Médecine translationnelle, UR3072, Strasbourg Strasbourg, France; ${ }^{3}$ University of Strasbourg, Service de Physiologie et d'explorations fonctionnelles, Nouvel Hopital Civil, Fédération de Médecine Translationnelle, UR3072, Strasbourg, Strasbourg, France; ${ }^{4}$ Centre Hospitalie Universitaire, Strasbourg, Department of Rheumatology, Strasbourg, France

Background: Myositis are systemic autoimmune rare diseases characterized by muscle inflammation and weakness. Even though the signs of active disease have been resolved, myositis patients frequently present residual muscle weakness, decreased physical performance and sustained disability. This condition has been coined on the term "damage" (as opposed to "activity").

Sarcopenia is a frequent, progressive and generalized skeletal muscle disorder characterized by low muscle strength and mass leading to handicap, decreased quality of life and increased mortality.

Prevalence and significance of sarcopenia in myositis patients has never been reported.

Objectives: To study sarcopenia in myositis patients with low or no disease activity.

Methods: Adult myositis patients (2017 ACR/EULAR criteria), with disease duration greater than 12 months, creatine kinase serum level (CK) less than $500 \mathrm{U} / \mathrm{I}$ stable medication for 6 months were enrolled. Patients with inclusion body myositis were excluded. Total (LM) and appendicular (ALM) muscle mass were measured using dual-energy X-ray absorptiometry (DXA, Hologic) and muscle grip strength was measured using Jamar dynamometer. Sarcopenia was defined according to the EWGSOP2 consensus. ${ }^{1}$

Results: 29 patients ( 20 female, $68.9 \%$ ), with a median age of 61 years (50.5 71 ) were enrolled. They suffered from dermatomyositis ( $D M, n=4)$, immune-mediated necrotizing myopathy (IMNM), $n=8$ ), anti-synthetase syndrome (ASS, $n=9$ ), scleromyositis (SM, $n=8$ ) since 4.7 years (2.8-8.3). At the evaluation, muscle strength assessed with MMT-8 was 139/150 (136-147), MMT-12 was 210/220 (204-216) and CK were 131.5 U/I (105.5-202). Four patients (13.8\%) were sarcopenic. Sarcopenic patients were older (73.4 years $(66.2-80.5)$ vs 58.7 years (44.2-79.6), $p=0.03)$, with a longer disease duration (7.3 years (5.3-11.8) vs 4.3 $(2.7-8.3), p=0.1)$, longer time with increased CK (449 days (169.8-954) vs 255.5 (124-872.8)), higher maximum CK values (6000 U/l (2205-7000) vs 1636 (9004457)). They suffered from IMNM (2/4, 50\%); DM ( $n=1)$ and SM $(n=1)$, had more frequently disease-related cardiac involvement $(50 \%$ vs $4 \%, p=0.04)$, and tended to a longer steroid therapy duration (2.4 years $(0.8-5)$ vs $1.8(1.3-3.9), p=0.9)$ and a higher number of immunomodulatory drugs (2.5 (2-5.3) vs $2(2-3), p=0.3)$. At the evaluation, sarcopenic patients were globally weaker as highlighted by lower MMT-12 (201 (196.8-206.8) vs 213 (207-217.5), p=0.02). Head flexo-extensors and proximal upper muscles were especially weaker (respectively, $p=0.04$ and $p=0.03$ ). Muscle performance was also lower in sarcopenic patients as assessed by distance covered at 6 -minute walk test $(6 \mathrm{mWT}, \mathrm{p}=0.003)$ and number of squats in 30 seconds $(p=0.005)$. Time to drink a glass of water was significantly longer in sarcopenics $(p=0.04)$ even if any patient referred dysphagia Health assessment questionnaire score was greater $(1.4(0.8-2)$ vs $0.6(0.2-1)$ $p=0.04)$ indicating higher handicap. LM positively correlated with MMT-8 $(\rho=0.5$ $p=0.01)$ and MMT-12 $(\rho=0.5, p=0.004)$, with number of squats in 30 seconds $(\rho=0.7, p=0.0003)$ and $6 \mathrm{mWT}$ distance covered/lower limit $(\rho=0.5, p=0.01)$. Moreover, LM negatively correlated with time to drink a glass of water $(\rho=-0.6$, $\mathrm{p}=0.002)$.

Conclusion: Muscle mass measured by DXA is a relevant parameter for muscle damage and disability in myositis patients. Sarcopenic myositis patients represent a subgroup with important muscle damage and handicap.

\section{REFERENCES:}

[1] Cruz-Jentoft AJ et al. Sarcopenia: revised European consensus on definition and diagnosis. Age Ageing. 1 Jan 2019;48(1):16-31.

Disclosure of Interests: None declared

DOI: 10.1136/annrheumdis-2021-eular.3248

\section{POS0877 \\ THE EFFECT OF PLATELET INHIBITORS ON DIGITAL ULCERS IN SYSTEMIC SCLEROSIS - A DERIVATION} AND VALIDATION EUSTAR STUDY

A. Garaiman ${ }^{1}$, K. Steigmiller ${ }^{2}$, C. Gebhard ${ }^{3}$, C. Mihai ${ }^{1}$, R. Dobrota ${ }^{1}$, M. MatucciCerinic $^{4}$, J. Henes ${ }^{5}$, J. De Vries-Bouwstra ${ }^{6}$, V. Smith ${ }^{7}$, A. Doria ${ }^{8}$, Y. Allanore ${ }^{9}$, L. Dagna ${ }^{10}$, B. Anic ${ }^{11}$, C. Montecucco ${ }^{12}$, O. Kowal-Bielecka ${ }^{13}$, M. Martin ${ }^{14}$, Y. Tanaka ${ }^{15}$, A. M. Hoffmann-Vold ${ }^{16}$, U. Held ${ }^{2}$, O. Distler ${ }^{1}$, M. O. Becker ${ }^{1}$. ${ }^{1}$ Department of Rheumatology, University Hospital Zurich, University of Zurich, Zürich, Switzerland; ${ }^{2}$ Department of Biostatistics, at Epidemiology, Biostatistics and Prevention Institute, University of Zurich, Zurich, Switzerland; ${ }^{3}$ Department of Nuclear Medicine, University Hospital Zurich, University of Zurich, Zürich, Switzerland; ${ }^{4}$ Department of Experimental and Clinical Medicine, Division of Rheumatology, University of Florence, Scleroderma Unit, AOUC, Florence, Italy; ${ }^{5}$ Centre for Interdisciplinary Clinical Immunology, Rheumatology and Autoinflammatory Diseases and Department of Internal Medicine II (Hematology, Oncology, Immunology and Rheumatology), University Hospital Tuebingen, Tuebingen, Germany; ${ }^{6}$ Department of Rheumatology, Leiden University Medical Center, Leiden, Netherlands; ${ }^{7}$ Department of Internal Medicine, Ghent University, Belgium; Department of Rheumatology, Ghent University Hospital, Belgium; Unit for Molecular Immunology and Inflammation, VIB Inflammation Research Center (IRC), Ghent, Belgium; ${ }^{8}$ Rheumatology Unit, Department of Medicine, University of Padova, Padova, Italy; ${ }^{9}$ Department of Rheumatology A, Descartes University, APHP, Cochin Hospital, Paris, France ${ }^{10}$ Unit of Immunology, Rheumatology, 
Allergy and Rare Diseases (UnIRAR), IRCCS San Raffaele Scientific Institute, Milan, Italy; Vita-Salute San Raffaele University, Milan, Italy; ${ }^{11}$ Division of Clinical Immunology and Rheumatology, Department of Internal Medicine, University Hospital Centre Zagreb and University of Zagreb, School of Medicine, Zagreb, Croatia; ${ }^{12}$ Department of Rheumatology, Fondazione IRCCS Policlinico San Matteo, University of Pavia, Pavia, Italy; ${ }^{13}$ Department of Rheumatology and Internal Medicine, Medical University of Bialystok, Bialystok, Italy; ${ }^{14}$ Department of Internal Medicine, Poitiers University Hospital, Poitiers, France; ${ }^{15}$ The First Department of Internal Medicine, School of Medicine, University of Occupational and Environmental Health Japan, Kitakyushu, Japan; ${ }^{16}$ Department of

Rheumatology, Oslo University Hospital, Oslo, Norway

Background: Digital ulcers (DUs) affect half of the patients with systemic sclerosis (SSc) and can be complicated by gangrene and amputation. The direct involvement of platelets in the development of DUs has been suggested by in vitro studies, which encouraged physicians to consider platelet inhibitors as a therapeutic option in the management of DUs. However, until now, there is no clinical study to assess the efficacy of platelet inhibitors for DUs in SSc patients. Objectives: To demonstrate a possible relationship between treatment with platelet inhibitors and the occurrence of DUs at the next follow-up visit in patients with SSc.

Methods: This study used prospectively collected data from the European Scleroderma Trials and Research group (EUSTAR) registry. Patients fulfilling the 2013 ACR/EULAR SSc classification criteria with complete longitudinal data on the presence of DUs and platelet inhibitors were included in the analysis. Multiple imputation using a random forest algorithm was implemented to handle missing values.

The dataset was split into a derivation and validation cohort. To investigate the response for the binary dependent variable of DUs, a generalized linear mixed model (GLMM) was developed in the derivation cohort and validated using ROC analysis and Brier scores to address discrimination and calibration, respectively. Results: Of 3,463 patients (2,961 in the derivation cohort, 722 in the validation cohort), 453 had current DUs at the baseline and 245 were exposed to platelet inhibitors (table 1 ). Our GLMM revealed that the exposure to platelet inhibitors is associated with a reduced risk of DUs at the next follow up visit (OR $=0.33,95 \% \mathrm{Cl}=[0.13$ to 0.82$]$ ). Further factors associated with absence or presence of DUs at the next follow-up visit are shown in figure 1. This confirmed the previously identified risk factors for the presence of DUs, supporting the overall robustness and the validity of our model. The performance was evaluated by ROC curve analysis and showed an AUC = $97.97 \%(95 \% \mathrm{Cl}=[96.93 \%$ to $97.67 \%])$ for the derivation cohort and $\mathrm{AUC}=77.3 \%$ $(95 \% \mathrm{Cl}=[74.01 \%$ to $81.39 \%])$ for the validation cohort, respectively, showing an acceptable discrimination. The Brier score was 0.05 in the derivation cohort and 0.07 in the validation cohort, suggesting a good calibration of the model.

Conclusion: Our model, with acceptable discrimination and good calibration, suggests a positive treatment effect of platelet inhibitors on DUs in clinical practice.

Table 1. Baseline characteristics of patients before imputation

\begin{tabular}{llll}
\hline Characteristics & Overall & Derivation set & Validation set \\
\hline $\mathrm{n}$ & 3,463 & 2,691 & 772 \\
Age (median [IQR]) & $56.00[47.00,66.00]$ & $56.00[47.00,65.00]$ & $57.00[48.00$, \\
& & & $67.00]$ \\
Disease duration (median [IQR]) & $9.00[4.00,16.00]$ & $9.00[4.00,16.00]$ & $8.00[4.00$, \\
& & & $15.00]$ \\
Disease subset = Limited cutane- & $1562(65.2)$ & $1164(64.6)$ & $398(66.9)$ \\
$\quad$ ous SSc (\%) & & & \\
DUs (\%): Current & $453(13.1)$ & $378(14.0)$ & $75(9.7)$ \\
DUs (\%): Never & $1783(51.5)$ & $1326(49.3)$ & $457(59.2)$ \\
DUs (\%): Previously & $1227(35.4)$ & $987(36.7)$ & $240(31.1)$ \\
mRSS (median [IQR]) & $5.00[2.00,11.00]$ & $6.00[2.00,12.00]$ & $4.00[1.00$, \\
& & & $11.00]$ \\
Joint Contractures = Yes (\%) & $881(26.8)$ & $770(29.4)$ & $111(16.5)$ \\
LVEF (median [IQR]) & $62.00[60.00,65.00]$ & $60.00[60.00,65.00]$ & $65.00[60.00$, \\
& & & $67.00]$ \\
Dyspnea NYHA III and IV (\%) & $300(9.5)$ & $214(8.6)$ & $86(12.7)$ \\
Pulmonary hypertension =Yes (\%) & $244(10.7)$ & $200(11.3)$ & $44(8.4)$ \\
Lung fibrosis on HRCT =Yes (\%) & $685(46.6)$ & $600(47.7)$ & $85(39.7)$ \\
FVC \% predicted (median [IQR]) & $97.00[82.00,111.00]$ & $95.00[81.00,110.00]$ & $101.00[85.00$, \\
& & & $115.00]$ \\
Serum creatinine mg/dl (median & $0.70[0.60,0.90]$ & $0.70[0.60,0.90]$ & $0.70[0.70$, \\
$\quad$ [IQR]) & & & $0.90]$ \\
Anti-Scl-70 positive = Yes (\%) & $1147(33.1)$ & $958(35.6)$ & $189(24.5)$ \\
CRP elevation = Yes (\%) & $639(21.1)$ & $490(20.8)$ & $149(22.1)$ \\
Platelet inhibitors therapy =Yes (\%) & $245(7.1)$ & $206(7.7)$ & $39(5.1)$ \\
Oral anti-coagulants therapy =Yes (\%) $53(1.5)$ & $50(1.9)$ & $3(0.4)$ \\
\hline
\end{tabular}

Figure 1: The coefficient plot of the GLMM:

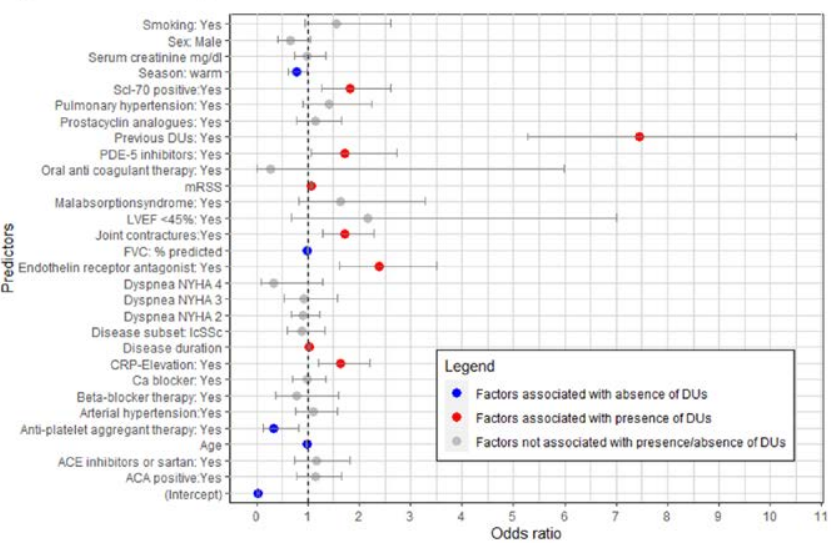

Disclosure of Interests: None declared

DOI: 10.1136/annrheumdis-2021-eular.3272

\section{POS0878 ULTRASOUND ASSESSMENT OF DERMAL THICKNESS AND SKIN STIFFNESS IN UNDIFFERENTIATED CONNECTIVE TISSUE DISEASE AT RISK FOR SYSTEMIC SCLEROSIS}

T. Santiago $^{1,2}$, M. Luis ${ }^{1}$, J. Lima ${ }^{2}$, C. Gaspar ${ }^{2}$, M. J. Salvador ${ }^{1,2}$, J. A. P. Da Silva ${ }^{1,2} .{ }^{1}$ Centro Hospitalar e Universitário de Coimbra, Rheumatology Department, Coimbra, Portugal; ${ }^{2}$ Faculty of Medicine, University of Coimbra, Institute for Clinical and Biomedical Research (iCBR), Coimbra, Portugal

Background: High-frequency ultrasound (HFUS) and shear-wave elastography (SWE) allow an objective assessment of skin involvement in systemic sclerosis (SSc) patients. ${ }^{1}$ Till now it has been applied to patients with established diagnosis. ${ }^{2,3}$ However, there is no data concerning its application in Undifferentiated Connective Tissue Disease at risk for SSc (UCTD-risk-SSc), i.e., patients with Raynaud's phenomenon and either SSc marker autoantibodies or typical capillaroscopic findings or both, not satisfying classification criteria for SSc. ${ }^{4}$

Objectives: To compare ultrasound-dermal thickness (DT) and skin stiffness, using high-frequency ultrasound and shear-wave elastography, in UCTD-risk-SSc and healthy controls.

Methods: Forty UCTD-risk-SSc patients and 40 age- and gender-matched healthy controls were included. Ultrasound-DT was measured using an $18 \mathrm{MHz}$ probe, and skin stiffness (i.e. shear-wave velocity values, SWV) using the VTIQ software with a $9 \mathrm{MHz}$ probe, at the 17 Rodnan skin sites. The mRSS score was, by definition, zero in all sites, both in cases and controls. Continuous data were expressed as the mean (SD), and Mann-Whitney $U$ test was performed to compare differences between the groups, as variables were not normally distributed. Associations between variables were analysed using the Spearman's correlation.

Results: SWV values were significantly higher in patients with UCTD-riskSSc compared with controls at the right and left hands, and in the right and left fingers (table 1). Higher values of ultrasound dermal-thickness were found in the fingers and hands bilaterally, although differences were only significantly at the hands, compared with healthy controls (table 1). There were no significant differences in the other Rodnan skin sites. There was no significant correlation between ultrasound-dermal thickness and stiffness at the same skin site.

Conclusion: This study provides the first evidence suggesting that ultrasound-DT and stiffness can discriminate patients with UCTD-risk-SSc from healthy controls. Prospective studies including a larger number of patients with different subsets of UCTD-risk-SSc are needed to investigate diagnostic and prognostic value of the ultrasound parameters in this group.

\section{REFERENCES:}

[1] Santiago T, et al. Arthritis Care Res (Hoboken). 2019;71:563-574.

[2] Hesselstrand R, et al. Rheumatology (Oxford). 2008;47:84-7.

[3] Flower $\mathrm{V}$ et al. jrheum.200234.

[4] Valentini, G., et al. Arthritis Care Res, 66: 1520-1527. 